\title{
Symposium on Biochemical and Clinical Aspects of Steroid Enzymology
}

The 5th meeting of the International Study Group for Steroid Hormones will be held in Rome from December 6-8, 1971. The principal theme of this Symposium will be "Biochemical and Clinical Aspects of Steroid Enzymology". Further details can be obtained from the Secretary of the Study Group: Prof. Carlo Conti, Istituto di Patologia Medica II, Policlinico Umberto 1, Universitá di Roma, Rome, Italy. 